\title{
SILENT AND SPOKEN ANIMATED SITUATIONAL MATERIALS: A PROCEDURE OF INNOVATIVE AND ETHICAL STANDARDS IN EDUCATIONAL IMPROVEMENT
}

\author{
Luma Ibrahim Al-barzenji \\ Prof. Dr. Department of English Language and Literature, Faculty of Education and Humanities \\ Diyala University, Iraq, Lumahh50@gmail.com
}

\begin{abstract}
The assessment of education achievements and levels of global competition are going over development all the time. To achieve this target, educational system seriously is looking forward to establish some procedures of how to improve their international standing. Among these targeting procedures is to grant the best teaching process levels that are used to receive preferable and powerful results. In the process of education, teaching could be influential when the purpose of teaching is directed to examine the potential use of animated learning situational materials. Animated materials are received as being an effective means of teaching messages and lessons that static materials could not offer. This in turn transforms the rigid student's understanding into a fully interactive performance. That way of presenting teaching materials comes up with story ideas which are always hard to make them much easier of virtual experiences. Educational problems could be solved when values and morals are seen out of restricted methods. Hence, building up a successful challenge through animation is the process of bringing life experience into reality shaped with color, dialogue, plot, characters, and setting through an animated situation. Thus, morals, ideals, and teaching messages might be taught within the topic of school assessment policy.
\end{abstract}

Keywords: Animated educational material, virtual experiences, self-expressio

\section{SECTION I: WHY ANIMATION}

Teachers and animation designers should take into consideration the best way of the integration between the written text and the visual illustration. Such a kind of integrated educational materials is more understandable than using other visual arts such as cards or dramatic scenes. Going further, silent animated stories, educational situations, and social subjects provide other successful opportunities to learning improvement. Activities of analyzing, discussing, reacting, and interpreting encourage student's self-creativity and self-expression. Students in silent animated educational situations are able to use more than one skill starting from their excitement to observe and analyze until the step of how to react by "with or against". Hence, animation functions student's multiple abilities to perform mental, psychological, and social processes than any classical static subject material. Additionally, animated materials, silent or spoken, improve student's imagination to visualize specific educational concepts that require a particular 
IJAEDU- International E-Journal of Advances in Education, Vol. V, Issue 13, April, 2019

understanding.

Animated educational material is more than a technique. It supports students with a sense of innovative technologies to create a world that develops successfully a system of cause and effect (moral, ethical, and educational).

While at first glance, animation may be evaluated as means to display numerous messages directed to kids or young children, while many of these messages are functioned to depict deeper meanings appreciated by an adult audience. Themes of the animated educational materials are mostly inspiring to educate the viewers, regardless of specific ages.

In modern educational systems, screenplay presents varieties of learning targets that aim to come close the messages and lessons behind. Viewers and students of different levels can communicate with these virtual values emotionally, analytically, and re-actionably whenever they feel they are watching a good piece of displaying. For example, themes like "change" starts from self -inside, spiritual beauty is a value, listening before speaking, are effectively proved or disproved if they are watching these values as animated goals. What makes themes important is the screenplay when the latter displays the plot, hero, villain, actions, dialogue, and setting.

As the very best example of animated subject effect, Disney films often present mythology and animated characters to depict certain values and to deliver a message of the importance of being true, faithful, and loyal as explicit themes in Walt Disney's Moana. Such animated story acting movies support wonderful pieces of music accompanied to the storytelling that are used in crucial bits of the story events. The voice acting works together with the plot and sub-plot to develop the theme. Moreover, animated topics offer interesting space of humor that keeps the screenplay fresh throughout.

Through a thematic study of a certain educational target, self-assertion, self-creativity, and self-definition may be fostered and encouraged refreshingly by animated situation found in various plots. Such targets can be given whatever the teacher like without the rigid way of instructing words or phrases. Thus, morals of how to behave confidently and truly by spoken instructions will not be matter too much because words mostly become exceedingly heavy weight.

\section{SECTION II: ANIMATION IN ARTS AND CULTURE}

For both educational and entertaining purposes, animated movies can even be life changing. Animation is important in artistic, cultural, and economic senses (Wells 2008). From art point of view, the design of animation gathers the art of storytelling, music, sound effects, color, etc., while culturally, animated movies proved the difficulty of living a life without a sense of fantasy of the animated characters that fill our imagination. Economically, animation provides opportunities for producers to employ hundreds of artists, animators, and technicians. Besides, animation is a technological style that to share, promote, and educate a variety of audiences. It is a perfect way of communicating concepts of all variety of audiences. It allows bringing to life ideas that may be prohibited or impossible with true live atmosphere. For example, how a lion talks to human or competes with him? Without a considerable risk, animation could do that interestingly and educationally. Furthermore, an imaginative challenge brings a sense of creativity to promote a real challenge that leads to life fantasies. It is deeply connected with human brains from childhood to depict unrealistic events and it easily teaches lessons in an art-form like. Thus, it is a mix of creativity, meaning, morals, imagination, ethics, colors, and plot. Teachers implement animation in their teaching strategy to help giving opportunities to understand and involve positively in the learning process.

Animation is used to spread social messages of thoughts and behavior assessment, interactive social access, or developing mental and psychological interests. In teaching, animated educational subject fills the imagination with wonder. Students may find a great interest to follow the positive behaviors of animated characters and keep wondering about the best ways of life adjustment. Students also would believe that in animation people would be pulled to another sort of life encouraging them to suspend disbelief. In this way, teachers and students will involve in what is so-called Mystery Science when both ideas are matured and scientific. Here, teachers allow their students to communicate with abstract and to engage in more idealistic concepts.

In silent animated stories, picture motion helps to uplift student's ability to analyze, interpret, and to imagine what could be said in spoken action ( Heuer 34). Personality traits are developed when silent performance is working on how to expand the learner's mental part. In a certain degree, teachers in the process of adapting new learning technologies are inspired by silent animation more than ever. Visual animation with silence encourages viewers' awareness in many ways. It raises interest of how to interpret successfully the targeting ideas of the movie and how to react in verbal or spoken words. Hence, teachers should come to terms that 
doing or reacting this without words can be incredibly powerful because students may be asked to re-act silently or in wordless action while explaining the watched scenes. For example, in Dolby, the silent animated short movie shows how powerful silent animation plot could be presented today. Moreover, such a kind of movie celebrates how technology elevates the trend of silence in presenting moral and educational messages.

Sound effects and music add a successful access to the silent animated excitement. Music of silent movie inspires students to analyze and elaborate a good interpretation by fostering their emotion to be excited without the use of words.

Through the process of technological adaptation, imagination should be uplifted when stimulating ideas are captured. This could be happened when animated videos are accompanied by sound effects of wordless action. Consequently, this overwhelms the viewers and helps them to focus on the positive messages and ethics. It creates good video explainers and clever thinkers who think of creative ideas.

\section{REFERENCE LIST}

Heuer, Richards. Jr (1999). Psychology of Inrelligence, Analysis, USA

Wells, Paul and Johnny Hardstaff (2008). Re-Imagining Animation: The Changing Face of the Moving Image. New York: Watson-Guptill Publications, 34 